\title{
ON TWO-GENERATOR DISCRETE GROUPS OF MÖBIUS TRANSFORMATIONS
}

\author{
DELIN TAN
}

(Communicated by John B. Conway)

\begin{abstract}
Assume that Möbius transformations $f$ and $g$ generate a discrete group. We obtain the following generalizations of Jørgensen's inequalities. If $\operatorname{tr}\left(f g f^{-1} g^{-1}\right) \neq 1$, then $\left|\operatorname{tr}^{2}(f)-2\right|+\left|\operatorname{tr}\left(f g f^{-1} g^{-1}\right)-1\right| \geq 1$. If $\operatorname{tr}\left(f g f^{-1} g^{-1}\right)=1$, then either $\operatorname{tr}^{2}(f)=2$ or $\operatorname{tr}^{2}(f)=1$ or $\left|\operatorname{tr}^{2}(f)-2\right|>\frac{1}{2}$ and $\left|\operatorname{tr}^{2}(f)-1\right|>\frac{1}{2}$. If $\operatorname{tr}^{2}(f) \neq 1$, then $\left|\operatorname{tr}^{2}(f)-1\right|+\left|\operatorname{tr}\left(f g f^{-1} g^{-1}\right)\right| \geq 1$. If $\operatorname{tr}^{2}(f)=1$ then either $\operatorname{tr}\left(f g f^{-1} g^{-1}\right)=0$ or $\operatorname{tr}\left(f g f^{-1} g^{-1}\right)=1$ or $\left|\operatorname{tr}\left(f g f^{-1} g^{-1}\right)\right|>\frac{1}{2}$ and $\left|\operatorname{tr}\left(f g f^{-1} g^{-1}\right)-1\right|>\frac{1}{2}$.
\end{abstract}

\section{INTRODUCTION}

Let $f$ and $g$ be two Möbius transformations that generate a nonelementary discrete group. Then they satisfy Jørgensen's well-known inequality [5]:

$$
\left|\operatorname{tr}^{2}(f)-4\right|+\left|\operatorname{tr}\left(f g f^{-1} g^{-1}\right)-2\right| \geq 1 .
$$

It is interesting to generalize Jørgensen's inequality. There are many papers concerning such generalizations, for example, those published by Brooks and Matelski [2], Gilman [4] and Rosenberger [9]. These results suggest the following question: Do there exist other constants $a$ and $b$ such that

$$
\left|\operatorname{tr}^{2}(f)-a\right|+\left|\operatorname{tr}\left(f g f^{-1} g^{-1}\right)-b\right| \geq 1 ?
$$

In this paper, by refining the Jørgensen's Lie product technique, we obtain several inequalities of this kind for certain discrete groups. In fact, we establish the following results:

Theorem 1. Suppose that the Möbius transformations $f$ and $g$ generate a discrete group. If $\operatorname{tr}\left(f g f^{-1} g^{-1}\right) \neq 1$, then

$$
\left|\operatorname{tr}^{2}(f)-2\right|+\left|\operatorname{tr}\left(f g f^{-1} g^{-1}\right)-1\right| \geq 1 ;
$$

if $\operatorname{tr}\left(f g f^{-1} g^{-1}\right)=1$ and $\operatorname{tr}^{2}(f) \neq 2$, then

$$
\left|\operatorname{tr}^{2}(f)-2\right|>\frac{1}{2} \text {. }
$$

Received by the editors January 26, 1988, and in revised form November 18, 1988.

1980 Mathematics Subject Classification (1985 Revision). Primary 30F40, Secondary 20H10. 
Theorem 2. Suppose that the Möbius transformations $f$ and $g$ generate a discrete group. If $\operatorname{tr}^{2}(f) \neq 1$, then

$$
\left|\operatorname{tr}^{2}(f)-1\right|+\left|\operatorname{tr}\left(f g f^{-1} g^{-1}\right)\right| \geq 1 ;
$$

if $\operatorname{tr}^{2}(f)=1$, then

$$
\left|\operatorname{tr}\left(f g f^{-1} g^{-1}\right)\right|>\frac{1}{2} \quad \text { or } \quad \operatorname{tr}\left(f g f^{-1} g^{-1}\right)=0 ;
$$

and

$$
\left|\operatorname{tr}\left(f g f^{-1} g^{-1}\right)-1\right|>\frac{1}{2} \quad \text { or } \operatorname{tr}\left(f g f^{-1} g^{-1}\right)=1 .
$$

Theorem 3. Suppose that the Möbius transformations $f$ and $g$ generate a discrete group. If $\operatorname{tr}\left(f g f^{-1} g^{-1}\right) \neq 1$, then

$$
\left|\operatorname{tr}^{2}(f)-\operatorname{tr}\left(f g f^{-1} g^{-1}\right)\right|+\left|\operatorname{tr}\left(f g f^{-1} g^{-1}\right)-1\right| \geq 1 ;
$$

if $\operatorname{tr}\left(f g f^{-1} g^{-1}\right)=1$ and $\operatorname{tr}^{2}(f) \neq 1$, then

$$
\left|\operatorname{tr}^{2}(f)-1\right|>\frac{1}{2} \text {. }
$$

Remark 1. If we take

$$
f(z)=i z, \quad g(z)=2 z,
$$

then the lower bounds of (3) and (8) are attained. We see also (5) is sharp by taking

$$
f(z)=-z, \quad g(z)=\frac{z-1}{z+1} .
$$

The recent work of Maskit in response to a question of Gehring and Martin (see [3]) shows that inequalities (3), (5) and (8) are sharp for nonelementary groups [8]. However, the lower-bound halves of (4), (6), (7) and (9) are not the best possible. In fact, the above theorems are equivalent under the Lie product transformation.

Remark 2. Take the discrete group $G_{1}$ generated by

$$
f(z)=i z, \quad g(z)=\frac{z-1}{z+1}
$$

the discrete group $G_{2}$ generated by

$$
f(z)=\frac{z-i}{z+i}, \quad g(z)=-\frac{z-i}{z+i}
$$

and the discrete group $G_{3}$ generated by

$$
f(z)=\frac{z-i}{z+i}, \quad g(z)=\frac{i z+1}{z+i} .
$$

The group $G_{i}$ shows that hypothesis for Theorem $i$ is necessary.

Remark 3. Gehring and Martin have worked on the same problem and have established a general result which contains many inequalities including (3) and (8) as well as Jørgensen's inequality [3].

\section{ProOF OF THE THEOREMS}

The proof is based on the following lemmas: 
Lemma 1. Suppose that the Möbius transformations $f$ and $g$ generate a discrete group. If $\operatorname{tr}(f) \neq 0$ and $\operatorname{tr}\left(f g f g^{-1}\right) \neq 2$, then

$$
\left|\operatorname{tr}^{2}(f)\right|+\left|\operatorname{tr}\left(f g f^{-1} g^{-1}\right)+2\right| \geq 1 \text {. }
$$

Lemma 2. Suppose that the Möbius transformations $f$ and $g$ generate a discrete group. If $\operatorname{tr}\left(f g f g^{-1}\right) \neq 1$, then

$$
\left|\operatorname{tr}^{2}(f)-2\right|+\left|\operatorname{tr}\left(f g f g^{-1}\right)-1\right| \geq 1 ;
$$

if $\operatorname{tr}\left(f g f g^{-1}\right)=1$ and $\operatorname{tr}^{2}(f) \neq 2$, then

$$
\left|\operatorname{tr}^{2}(f)-2\right|>\frac{1}{2} \text {. }
$$

We will prove these lemmas in $\S 3$. In this section we use Lemma 2 to prove Theorems 1-3.

Let the matrices $A$ and $B$ represent $f$ and $g$ in $S L(2, C)$ respectively. If $\operatorname{tr}\left(A B A^{-1} B^{-1}\right)=2$, then there is nothing to prove. Next we suppose that $\operatorname{tr}\left(A B A^{-1} B^{-1}\right) \neq 2$. Let $\phi=A B-B A$ be the Lie product of $A$ and $B$, then $\phi$ is elliptic of order 2 and conjugates $A$ and $B$ to their inverses. The group $\langle A, B\rangle$ has index at most two in the group $\langle A, B, \phi\rangle$ and thus both groups are simultaneously discrete or nondiscrete. This is Jørgensen's Lie product construction [6 and 7].

Now applying Lemma 2 to $A$ and $B \phi$ yields Theorem 1 .

Notice that if we regard $\phi$ as a matrix, then $\phi^{2}=-I$. If we take $F=A B \phi$ and $G=A^{-1}$, then

$$
\begin{gathered}
\operatorname{tr}^{2}(F)=\operatorname{tr}(A B \phi)^{2}+2=-\operatorname{tr}\left(A B A^{-1} B^{-1}\right)+2, \\
\operatorname{tr}\left(F G F^{-1} G^{-1}\right)=\operatorname{tr}\left(A B A B^{-1}\right), \\
\operatorname{tr}\left(F G F G^{-1}\right)=-\operatorname{tr}\left(A^{2}\right)=-\operatorname{tr}^{2}(A)+2 .
\end{gathered}
$$

So applying Lemma 2 to $A B \phi$ and $A^{-1}$ yields (5) and (6), while applying (6) to $A$ and $B \phi$ yields (7). Then applying Theorem 1 to $A B \phi$ and $A^{-1}$, we have: if $\operatorname{tr}\left(A B A^{-1} B^{-1}\right) \neq 1$, then

$$
\left|\operatorname{tr}\left(A B A^{-1} B^{-1}\right)\right|+\left|\operatorname{tr}\left(A B A B^{-1}\right)-1\right| \geq 1 ;
$$

if $\operatorname{tr}\left(A B A B^{-1}\right)=1$ and $\operatorname{tr}\left(A B A^{-1} B^{-1}\right) \neq 0$, then

$$
\left|\operatorname{tr}\left(A B A^{-1} B^{-1}\right)\right|>\frac{1}{2} \text {. }
$$

Replacing $A$ and $B$ by $A$ and $B \phi$ in (18) and (19), we get Theorem 3.

If we continue the same trick, we come back to (3), (5), or (15).

\section{Proof of the lemmas}

Simply applying the Lie product extension with $A B \phi$ and $A^{-1}$ to Jørgensen's inequality in [7] yields Lemma 1. 
For the proof of Lemma 2, we need only to consider the case where $f$ is elliptic or loxodromic. Let $f$ and $g$ be represented respectively by the matrices $A$ and $B$ in $S L(2, C)$. The inequalities are conjugacy invariant and so we can assume $A$ is of the form

$$
A=\left(\begin{array}{cc}
u & 0 \\
0 & \frac{1}{u}
\end{array}\right) \quad(u \neq-1,0,1)
$$

and

$$
B=\left(\begin{array}{ll}
a & b \\
c & d
\end{array}\right) \quad(a d-b c=1)
$$

Then

$$
\operatorname{tr}^{2}(A)=(u+1 / u)^{2}, \operatorname{tr}\left(A B A B^{-1}\right)=a d(u-1 / u)^{2}+2
$$

and

$$
\operatorname{tr}\left(A B A^{-1} B^{-1}\right)=u^{2}+1 / u^{2}-a d(u-1 / u)^{2} .
$$

Now we suppose that $\operatorname{tr}\left(A B A B^{-1}\right) \neq 1$ and (15) is false. Then

$$
\begin{aligned}
r & =\left|\operatorname{tr}^{2}(A)-2\right|+\left|\operatorname{tr}\left(A B A B^{-1}\right)-1\right| \\
& =\left|u^{2}+1 / u^{2}\right|+\left|1+a d(u-1 / u)^{2}\right|<1 .
\end{aligned}
$$

Let

$$
\begin{gathered}
B_{1}=B, \\
B_{n+1}=A_{n} B_{n} A_{n}^{-1} B_{n}^{-1}=\left(\begin{array}{ll}
a_{n+1} & b_{n+1} \\
c_{n+1} & d_{n+1}
\end{array}\right) \quad(n=1,2,3, \ldots) .
\end{gathered}
$$

Then we have

$$
\begin{aligned}
& a_{n+1}=a_{n} d_{n}\left(1-u^{2}\right)+u^{2}, \\
& b_{n+1}=a_{n} b_{n}\left(u^{2}-1\right), \\
& c_{n+1}=c_{n} d_{n}\left(\frac{1}{u^{2}}-1\right), \\
& d_{n+1}=a_{n} d_{n}\left(1-1 / u^{2}\right)+1 / u^{2} .
\end{aligned}
$$

Hence

(23) $1+a_{n+1} d_{n+1}(u-1 / u)^{2}=\left[1+a_{n} d_{n}(u-1 / u)^{2}\right]\left[u^{2}+1 / u^{2}-1-a_{n} d_{n}(u-1 / u)^{2}\right]$.

Let $t=u^{2}+1 / u^{2}$ and $z_{n}=1+a_{n} d_{n}(u-1 / u)^{2}$; then (23) becomes

$$
z_{n+1}=z_{n}\left(t-z_{n}\right) \text {. }
$$

So

$$
\left|z_{2}\right| \leq\left|z_{1}\right|\left(|t|+\left|z_{1}\right|\right)=\left|z_{1}\right| r .
$$

Then by induction, we have

$$
\left|z_{n+1}\right| \leq\left|z_{n}\right| r \leq\left|z_{1}\right| r^{n} .
$$


Observe that $z_{n}$ tends to zero as $n$ tends to $\infty$. So from (22)

$$
\begin{aligned}
& a_{n+1}=\frac{u^{2}\left(u^{2}-z_{n}\right)}{u^{2}-1} \rightarrow \frac{u^{4}}{u^{2}-1}(n \rightarrow \infty), \\
& d_{n+1}=\frac{\left(u^{2} z_{n}-1\right)}{u^{2}\left(u^{2}-1\right)} \rightarrow \frac{-1}{u^{2}\left(u^{2}-1\right)}(n \rightarrow \infty) .
\end{aligned}
$$

Now we consider two cases.

Case 1. Suppose that $z_{n} \neq 0$ for all $n$. Then (26) implies that the $z_{n}$ are distinct and hence that the same is true of $a_{n}$ and $d_{n}$.

If $f$ is elliptic, then $|u|=1$. Therefore for some constant $K$,

$$
\left|b_{n+2}\right|=\left|u^{2}-z_{n}\right|\left|b_{n+1}\right| \leq\left(1+K r^{n}\right)\left|b_{n+1}\right|
$$

and

$$
\left|c_{n+2}\right|=\left|1-u^{2} z_{n}\right|\left|c_{n+1}\right| \leq\left(1+K r^{n}\right)\left|c_{n+1}\right|
$$

Hence the sequences $\left\{b_{n}\right\},\left\{c_{n}\right\}$ are bounded.

If $f$ is loxodromic, then $|u| \neq 1$. Notice that the distinctness of $\left\{z_{n}\right\}$ implies for any $n, b_{n} \neq 0$. So we can choose an integer $k=k(n)$ such that

$$
1 \leq\left|u^{2 k} b_{n}\right| \leq\left|u^{2}\right|+\left|1 / u^{2}\right|
$$

Thus

has bounded entries.

$$
A^{k} B_{n} A^{-k}=\left(\begin{array}{cc}
a_{n} & u^{2 k} b_{n} \\
u^{-2 k} c_{n} & d_{n}
\end{array}\right)
$$

Hence we can find a subsequence of $\langle A, B\rangle$ which is distinct and bounded. This contradicts discreteness.

The above argument explicitly follows Jørgensen. (Also see [1].)

Case 2. Suppose that $z_{n}=0$ for some $n$.

The hypothesis $\operatorname{tr}\left(f g f g^{-1}\right) \neq 1$ means $z_{1} \neq 0$. From (24) we can find an integer $N$ such that

$$
z_{n}=0 \text { for } n>N \text { and } z_{n} \neq 0 \text { for } n \leq N
$$

So

$$
t=z_{N} \neq 0 \text { and }|t|=\frac{1}{2}\left(|t|+\left|z_{N}\right|\right) \leq \frac{1}{2}\left(|t|+\left|z_{1}\right|\right)<\frac{1}{2} \text {. }
$$

Also

$$
a_{n}=\frac{u^{4}}{u^{2}-1} \quad \text { and } d_{n}=\frac{-1}{u^{2}\left(u^{2}-1\right)} \text { for } n>N+1
$$

Thus we get a matrix $M$ in $\langle A, B\rangle$ of the form

$$
M=\left(\begin{array}{cc}
\frac{u^{4}}{u^{2}-1} & * \\
* & \frac{-1}{u^{2}\left(u^{2}-1\right)}
\end{array}\right)
$$


By computation

$$
M^{2}=\left(\begin{array}{cc}
\frac{u^{6}+u^{4}+1}{u^{2}-1} & * \\
* & -\frac{u^{6}+u^{2}+1}{u^{4}\left(u^{2}-1\right)}
\end{array}\right) .
$$

Now let $B_{1}$ be replaced by $M^{2}$ and return to the iteration of $B_{n}$. Let $z_{n}$ be defined as above corresponding to the new sequence. Then

$$
\begin{aligned}
z_{1} & =1+a_{1} d_{1}(u-1 / u)^{2} \\
& =1-\frac{\left(u^{6}+u^{4}+1\right)\left(u^{6}+u^{2}+1\right)}{u^{6}} \\
& =-t\left(t^{2}+t-2\right)
\end{aligned}
$$

and

$$
z_{2}=z_{1}\left(t-z_{1}\right)=-t^{2}(t-1)(t+2)\left(t^{2}+t-1\right)
$$

Let

$$
D_{1}=\left\{t: 0<|t| \leq \frac{1}{2} \text { and }\left|t(t-1)(t+2)\left(t^{2}+t-1\right)\right|<1\right\} .
$$

If $t \in D_{1}$, we have $0<\left|z_{2}\right|<|t|$. From (24)

$$
\left|z_{n}\right|\left(|t|-\left|z_{n}\right|\right) \leq\left|z_{n+1}\right| \leq\left|z_{n}\right|\left(|t|+\left|z_{n}\right|\right) \text {. }
$$

By induction we get

$$
0<\left|z_{n}\right| \leq\left|z_{2}\right|<|t| \text { and }|t|+\left|z_{n}\right| \leq|t|+\left|z_{2}\right|<1 \text { for } n \geq 2 .
$$

So we can go back to the case 1 . That means if $t \in D_{1}$, then $\langle A, B\rangle$ is not discrete.

By simple computation, we see that

$$
D_{1} \supset\left\{t: 0<|t| \leq \frac{1}{2} \text { and }-\pi / 4 \leq \arg (t) \leq \pi / 4\right\} \text {. }
$$

Now from (33)

$$
\left(A^{-1} M\right)^{2}=\left(\begin{array}{cc}
\frac{u^{4}+1}{u^{2}-1} & * \\
* & -\frac{u^{4}+1}{u^{2}\left(u^{2}-1\right)}
\end{array}\right) \text {. }
$$

We redefine the matrix $B$ to be

$$
B=\left(\begin{array}{ll}
a & b \\
c & d
\end{array}\right)=A^{3}\left(A^{-1} M\right)^{2}=\left(\begin{array}{cc}
\frac{u^{3}\left(u^{4}+1\right)}{u^{2}-1} & * \\
* & -\frac{u^{4}+1}{u^{5}\left(u^{2}-1\right)}
\end{array}\right) .
$$

Then

$$
\operatorname{tr}^{2}(B)=(a+d)^{2}=t^{4}(t+2),
$$

and

$$
a d=\frac{-t}{t-2} .
$$

If we set $F=A^{2}$, then from (20)

$$
\operatorname{tr}\left(B F B^{-1} F^{-1}\right)=\operatorname{tr}\left(F B F^{-1} B^{-1}\right)=t^{3}+3 t^{2}-2 .
$$


When $0<|t| \leq \frac{1}{2}$

$$
\operatorname{tr}^{2}(B) \neq 0 \text { and } \operatorname{tr}\left(B F B F^{-1}\right)=t^{5}+2 t^{4}-t^{3}-3 t^{2}+2 \neq 2 .
$$

So applying Lemma 1 to $B$ and $F$ yields

$$
\left|\operatorname{tr}^{2}(B)\right|+\left|\operatorname{tr}\left(B F B^{-1} F^{-1}\right)+2\right| \geq 1 .
$$

Thus

$$
\left|t^{4}(t+2)\right|+\left|t^{3}+3 t^{2}\right| \geq 1
$$

Let

$$
D_{2}=\left\{t: 0<|t| \leq \frac{1}{2} \text { and }\left|t^{4}(t+2)\right|+\left|t^{3}+3 t^{2}\right|<1\right\} .
$$

If $t \in D_{2}$, then $\langle A, B\rangle$ is not discrete.

By simple computation, we see that

$$
D_{2} \supset\left\{t: 0<|t| \leq \frac{1}{2} \text { and } \pi / 4 \leq \arg (t) \leq 7 \pi / 4\right\} \text {. }
$$

Combining (39) with (49), we conclude that $\langle A, B\rangle$ is not discrete. So we get (15).

If $\operatorname{tr}\left(A B A B^{-1}\right)=1$, then $z_{1}=0$. The same argument yields $t \notin D_{1} \cup D_{2}$. So $t=0$ or $|t|>\frac{1}{2}$ and (16) follows. This finishes the proof of the Lemma 2 .

\section{Conjecture}

The results established suggest the following very interesting question: For what kinds of discrete groups $\langle f, g\rangle$ will the left-hand sides of inequalities (1), (3), (5), or (14) be zero?

The left-hand side of (1) is zero if $f(z)=z+1, g(z)=z+\tau$ ( $\tau$ nonreal). Here $f$ and $g$ are both parabolic and $\langle f, g\rangle$ has signature $(1,0)$.

The left-hand side of (3) is zero if $f(z)=i z, g(z)=(z-1) /(z+1)$. Here $f$ and $g$ are both of order 4 and $\langle f, g\rangle$ has signature $(0,3 ; 2,3,4)$.

The left-hand side of $(5)$ is zero if $f(z)=(z-i) /(z+i), g(z)=$ $-(z-i) /(z+i)$. Here $f$ and $g$ are both of order 3 and $\langle f, g\rangle$ has signature $(0,3 ; 2,3,3)$.

Finally the left-hand side of (14) is zero if $f(z)=-z, g(z)=1 / z$. Here $f$ and $g$ are both of order 2 and $\langle f, g\rangle$ has signature $(0,3 ; 2,2,2)$.

In each of the four cases discussed above, the group $\langle f, g\rangle$ is elementary. From these observations, we are led to make the following conjecture:

Let $\langle F, G\rangle$ be an elementary discrete group with $\operatorname{tr}(F)=\operatorname{tr}(G)$. If $\langle f, g\rangle$ is discrete, then

$$
\left|\operatorname{tr}^{2}(f)-\operatorname{tr}^{2}(F)\right|+\left|\operatorname{tr}\left(f g f^{-1} g^{-1}\right)-\operatorname{tr}\left(F G F^{-1} G^{-1}\right)\right| \geq 1,
$$

provided that some appropriate auxiliary conditions are satisfied.

For example, if we take $F(z)=-z, G(z)=-z+1$, the signature of $\langle F, G\rangle$ is $(0,3 ; 2,2, \infty)$. Then $(50)$ will become

$$
\left|\operatorname{tr}^{2}(f)\right|+\left|\operatorname{tr}\left(f g f^{-1} g^{-1}\right)-2\right| \geq 1 \text {. }
$$


So far we can prove only the following: [10] If $\langle f, g\rangle$ is nonelementary discrete and $\operatorname{tr}(f) \neq 0$, then

$$
\left|\operatorname{tr}^{2}(f)\right|+\left|\operatorname{tr}\left(f g f^{-1} g^{-1}\right)-2\right|>2(\sqrt{2}-1)=0.828 \cdots .
$$

The accurate lower bound of (52) is unknown.

\section{ACKNOWLEDGMENTS}

I wish to express my heartfelt thanks to Professors Kra and Maskit for their careful reading of the manuscript, helpful conversation, and great encouragement during the preparation of this paper.

\section{REFERENCES}

1. A. F. Beardon, The geometry of discrete groups, Graduate Texts in Math., 91. Springer-Verlag, 1983.

2. R. Brooks and P. Matelski, The dynamics of two-generator subgroups of $\operatorname{PSL}(2, C)$, Ann. of Math. Stud. 97, Princeton Univ. Press, pp. 65-71.

3. F. W. Gehring and G. J. Martin, Discreteness in Kleinian groups and the iteration theory of quadratic mappings (to appear).

4. J. Gilman, A geometric approach to the hyperbolic Jorgensen inequality, Bull. Amer. Math. Soc. (N. S.) 16 (1987), no. 1, 91-92.

5. T. Jørgensen, On discrete groups of Möbius transformations, Amer. J. Math. 98 (1976), 737749.

6. Compact 3-manifolds of constant negative curvature fibering over the circle, Ann. of Math. 106 (1977), 61-72.

7. Comments on a discreteness condition for subgroups of $S L(2, C)$, Canad. J. Math. 31 (1979), 87-92.

8. B. Maskit, Some special 2-generator Kleinian groups, Proc. Amer. Math. Soc. (to appear).

9. G. Rosenberger, Eine Bemerkung zu einer Arbeit von T. Jørgensen, Math. Z. 165 (1979), 261-265.

10. D. Tan, On inequalities for discrete groups of Möbius transformations (to appear).

Mathematics Department, State University of New York at Stony Brook, STONY BROOK, NEW YORK 11794 